\section{What goes around...}
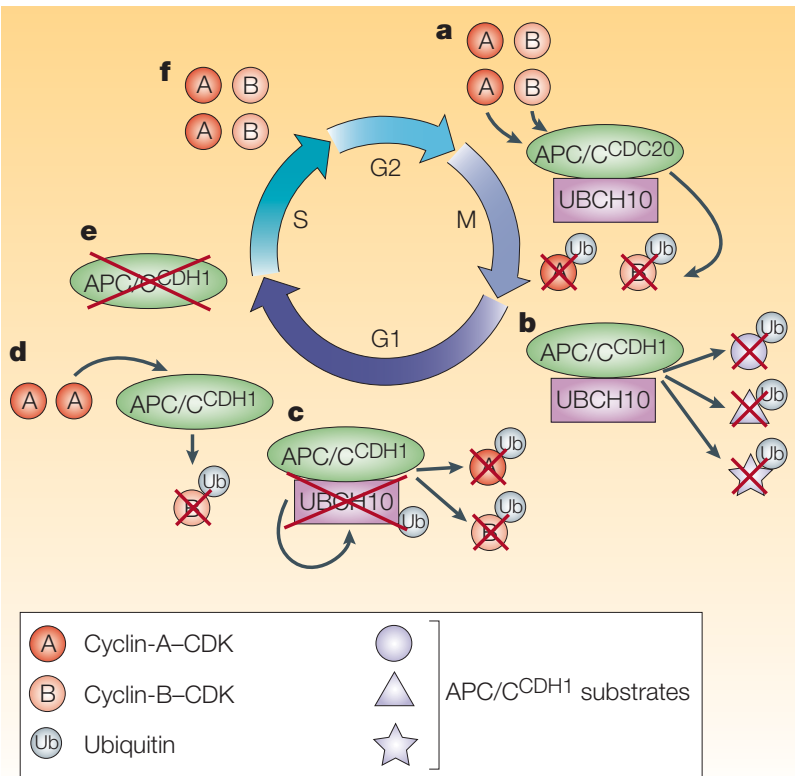

The transition from mitosis (M phase) to G1 phase is controlled by complex interactions between two key components of the cell-cycle machinery, the anaphase-promoting complex/cyclosome (APC/C) and cyclin A. The $\mathrm{APC} / \mathrm{C}$ is a multisubunit ubiquitin ligase that controls sister-chromatid separation and triggers mitotic exit through the ubiquitin-mediated degradation of proteins (including, in $\mathrm{M}$ phase, cyclin A). Cyclin A is an activator of the cell-cycle regulators $\mathrm{CDK} 1$ and $\mathrm{CDK} 2$ and, in late G1 phase, is also responsible for $\mathrm{APC} / \mathrm{C}$ inactivation.

How cyclin A can re-accumulate in the presence of active $\mathrm{APC} / \mathrm{C}$ during $\mathrm{G} 1$, and then inactivate the $\mathrm{APC} / \mathrm{C}$ to allow the re-accumulation of other mitotic cyclins during the DNA synthesis (S) and G2 phases, has been a puzzle. In Nature, Michael Rape and Marc Kirschner now report that they have solved the mystery, by studying the effects that different components and regulators of the APC/C have on the degradation of cyclin $\mathrm{A}$.

Initially, using extracts from synchronized HeLa cells, the authors showed that, whereas other APC/C substrates were consistently degraded throughout G1 phase, cyclin-A degradation gradually became blocked as cells progressed through G1. The degradation of cyclin A relied specifically on the presence of a threshold level of the ubiquitin-conjugating enzyme (E2) UBCH10, whereas other APC/C substrates were still degraded in the presence of another E2, UBCH5. In keeping with this, the authors showed that the level of UBCH10 (but not UBCH5) fluctuated during the cell cycle, remaining high during cyclin-A degradation and staying low when cyclin A was stable.

Intriguingly, it was then found that UBCH10 was autoubiquitylated before also being degraded by $\mathrm{APC} / \mathrm{C}^{\mathrm{CDH}} 1$ towards the end of $\mathrm{G} 1$. However, this process was hampered both by the slow rate of UBCH10 autoubiquitylation and by the presence of other APC/C substrates. In fact, the authors showed that, as long as other APC/C substrates are present in G1, UBCH10 preferentially ubiquitylates them, rather than itself, which effectively allows $\mathrm{UBCH} 10$ to orchestrate the timing of cyclin-A

\section{Details of the doorway}

The nuclear pore complex (NPC) functions as a doorway for the exchange of macromolecules between the nucleus and the cytoplasm, and a detailed view of this doorway is now revealed by Baumeister, Medalia and colleagues in Science.

The size of NPCs makes their structural characterization difficult, and previous structural studies have used isolated or detergent-extracted NPCs, which are susceptible to the loss of certain components and cargo. So, to get a more true-to-life view, Baumeister, Medalia and co-workers applied cryo-electron tomography (cryo-ET) to whole, transport-active Dictyostelium discoideum nuclei.

From the resulting tomograms, they extracted 267 volumes that contained an NPC and used averaging procedures to obtain an 8-9-nm-resolution, three- dimensional density map. In this map, the basic structural features of NPCs could be discerned: that is, eight cytoplasmic filaments that are attached to the cytoplasmic ring; the central, lumenal spoke ring; the central plug/transporter $(\mathrm{CP} / \mathrm{T})$ in the central channel; and the so-called nuclear basket (the nuclear ring and the distal ring that are connected by nuclear filaments).

However, the authors also noted several novel features - for example, the nuclear and cytoplasmic filaments appeared more bent and delicate than was previously reported, and the complex was less elongated along the nucleocytoplasmic axis. Furthermore, they found that the size, shape and position of the $\mathrm{CP} / \mathrm{T}$ in the NPCs varied. The composition and role of the $\mathrm{CP} / \mathrm{T}$ have remained unclear, but this observation indicates that it is composed, at least in part, of cargo complexes that are arrested in transit.

After further analysis, Baumeister, Medalia and colleagues noted that the NPCs were in one of two preferred states. In NPCs of the 'cytoplasmic-filament' class, the $\mathrm{CP} / \mathrm{T}$ is in the plane of the cytoplasmic ring. The cytoplasmic filaments are in a defined orientation and are connected to the CP/T by an elongated density. This structure therefore seems to show cytoplasmic filaments in a cargo-bound state. By contrast, in NPCs of the 'lumenal-ring' class, the $\mathrm{CP} / \mathrm{T}$ is in the plane of the lumenal spoke ring, and only the base of the cytoplasmic filaments can be seen. This structure therefore seems to show cytoplasmic filaments that have disengaged cargo and are free to move. Further differences in the organization of the lumenal spokes and the appearance of the nuclear basket could also be discerned between the NPC classes.

So, this work has shown that the $\mathrm{CP} / \mathrm{T}$ is essentially cargo in transit and that NPCs undergo significant structural rearrangements during cargo transit. In addition, although many questions remain, it has shown that "...the application of cryo-ET to transport-competent, intact nuclei holds great potential for a structural dissection of the key steps involved."

Rachel Smallridge

(2) References and links ORIGINAL RESEARCH PAPER Beck, M. et al. Nuclear pore complex structure and dynamics revealed by cryoelectron tomography. Science 306, 1387-1390 (2004) FURTHER READING Fahrenkrog, B. \& Aebi, U. The nuclear pore complex: nucleocytoplasmic transport and beyond. Nature Rev. Mol. Cell Biol. 4, 757-766 (2003) WEB SITE

Wolfgang Baumeister's laboratory: http://www.biochem.mpg.de/baumeister/ 